\title{
Si/SiGe-based edge-coupled photodiode with partially $p$-doped photoabsorption layer for high responsivity and high-power performance
}

\author{
J.-W. Shi, ${ }^{\text {a) }}$ P.-H. Chiu, F.-H. Huang, and Y.-S. Wu \\ Department of Electrical Engineering, National Central University, Taoyuan 320, Taiwan, \\ Republic of China \\ Ja-Yu Lu, C.-K. Sun, and C.-W. Liu \\ Department of Electrical Engineering, National Taiwan University, Taipei 106, Taiwan, Republic of China \\ and Graduate Institute of Electro-Optical Engineering, National Taiwan University, Taipei 106, \\ Taiwan, Republic of China \\ P.-S. Chen \\ Department of Materials Science and Engineering, Ming Hsin University of Science and Technology, \\ Hsinchu 304, Taiwan, Republic of China
}

(Received 5 December 2005; accepted 10 April 2006; published online 9 May 2006)

\begin{abstract}
We demonstrate a $\mathrm{Si} / \mathrm{SiG}$-based edge-coupled photodiode that can achieve high-speed, high output power, and high responsivity performance at a wavelength of $830 \mathrm{~nm}$ for application to short-reach fiber communication. We incorporate a $p$-type-doped $\mathrm{Si} / \mathrm{Si}_{0.5} \mathrm{Ge}_{0.5}$-based superlattice with a Si-based depletion layer to enhance the photoabsorption process and minimize the hole-trapping problem of the $\mathrm{Si} / \mathrm{SiGe}$ multiple quantum well. An extremely high bandwidth-efficiency product performance $(10 \mathrm{GHz}, 276 \%, 27.6 \mathrm{GHz})$ and high peak output voltage $(1.5 \mathrm{~V})$ have been achieved simultaneously by operating this device in the avalanche regime. (c) 2006 American Institute of Physics. [DOI: 10.1063/1.2202101]
\end{abstract}

Recently, several research groups have reported many kinds of Si-based high-speed photodetectors (PDs) for application in $850 \mathrm{~nm}$ short-reach fiber communication ${ }^{1}$ or visible single-photon detection. ${ }^{2}$ These include silicon-oninsulator (SOI) PDs, ${ }^{1,3}$ deep trench $p-i-n$ PDs, ${ }^{4}$ and metalsemiconductor-metal (MSM) PDs with a Si-based membrane. ${ }^{5}$ In order to further enhance the responsivity and bandwidth-efficiency product performance of Si-based PDs, the structures of resonant cavity enhanced (RCE) PDs with wafer-bonding mirrors ${ }^{6,7}$ and germanium $(\mathrm{Ge})$-on-SOI or Ge-on-Si PDs (Refs. 8 and 9) with high bandwidth-efficiency product performance have been demonstrated; however, their compatibility with the standard complementary metal oxide semiconductor (CMOS) or SiGe-based heterojunction bipolar transistor (HBT) process is still an open question ${ }^{3}$ due to their complex fabrication or material growth process. Besides for achieving the figures of merit (FOMs) of high bandwidth-efficiency product, the other major goal in the development of high-speed PDs is to attain high saturation output power. ${ }^{10}$ A PD with high responsivity and a wide dynamic range (saturation power) can eliminate the critical specifications of the next-stage CMOS-based widebandwidth electrical amplifier. In this letter, we demonstrate a $\mathrm{Si} / \mathrm{SiGe}$-based edge-coupled photodiode operated at a wavelength of $830 \mathrm{~nm}$. Incorporating a $p$-type-doped $\mathrm{Si} / \mathrm{SiGe}$-based superlattice (SL) with a depleted Si absorption layer in the photoabsorption region, this device achieves high peak output voltage and high bandwidth-efficiency product performance simultaneously.

Figures 1(a) and 1(b) show the conceptual band diagram and top view of the fabricated device, respectively. We adopted the structure of a traveling-wave photodiode ${ }^{11}$ to

\footnotetext{
a) Author to whom correspondence should be addressed; electronic mail: jwshi@ee.ncu.edu.tw
}

realize our device for high bandwidth-efficiency product performance. As shown in Fig. 1(b), the ridge optical waveguide structure can also minimize the long-tail problem, which originates from the substrate photocurrent, ${ }^{1,3,5-7}$ due to the confinement of the optical power in the surface epilayers. As shown in Fig. 1(a), the topmost $p^{+}$-doped $\mathrm{Si}$ layer and $n^{+}$-doped $\mathrm{Si}$ substrate serve as the cladding layers of the optical waveguide. The Si-based high-speed $p-i-n$ photodiode is usually operated in the avalanche regime to enhance its responsivity performance. ${ }^{3} \mathrm{We}$, thus, adopt a thin $\mathrm{Si}-$ based depletion layer $(150 \mathrm{~nm})$, which is sandwiched in between an $n^{+}$Si substrate and a $p$-type charge layer, to serve as the multiplication layer. Most of the external applied electric field is concentrated in this thin layer, and its avalanche breakdown effect benefits the responsivity performance. The core layer, which is sandwiched between two cladding layers, is composed of a $\mathrm{Si}_{0.5} \mathrm{Ge}_{0.5} / \mathrm{Si} \mathrm{SL}$ with a $150 \mathrm{~nm}$ thickness and a $p^{+}$heavily graded doped profile. Compared with directly growing bulk $\mathrm{Si}_{0.5} \mathrm{Ge}_{0.5}$ alloy on $\mathrm{Si}$ substrate, the

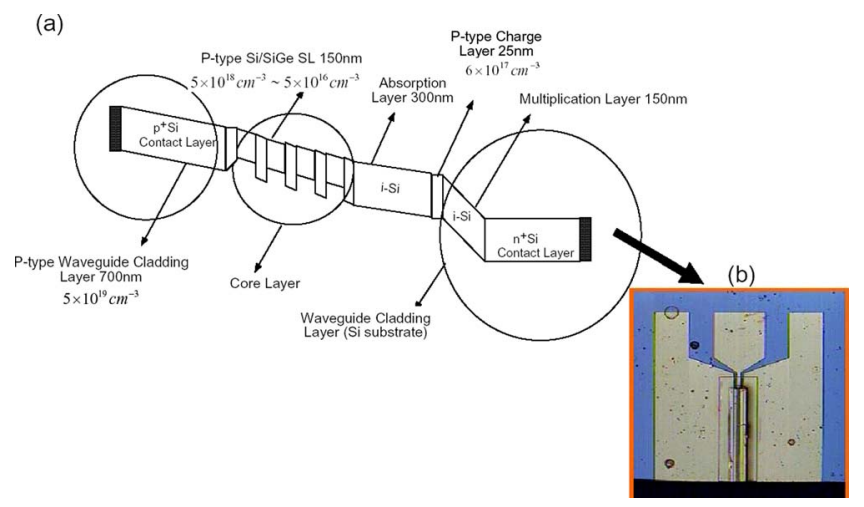

FIG. 1. (Color online) The conceptual band diagram (a) and top view (b) of the demonstrated $\mathrm{Si} / \mathrm{SiGe}$ photodiode. 


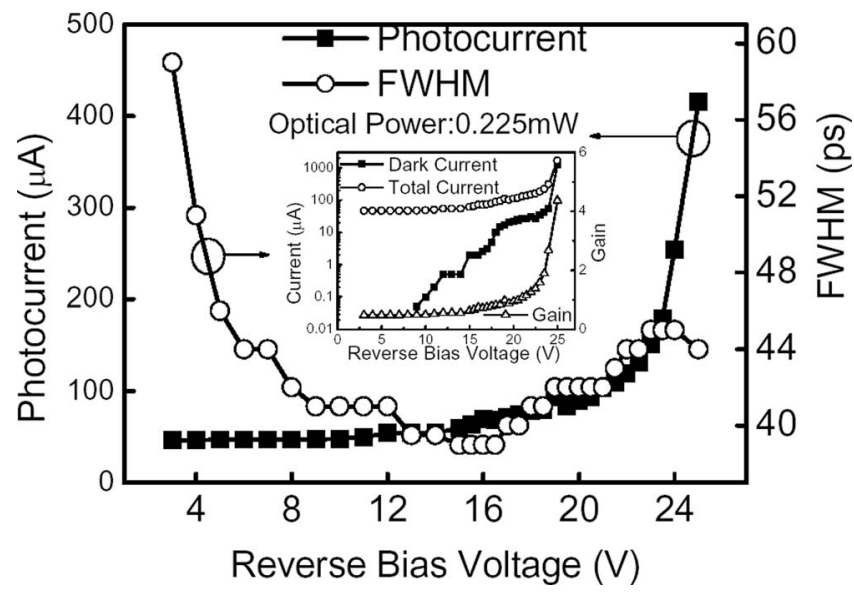

FIG. 2. (Color online) Photocurrent (close square) and FWHM (open circle) of the measured impulse responses vs the reverse bias voltages. The inset shows the measured total current (open circle), operation gain (open triangle) under avalanche operation, and the dark current (close square) vs the reverse bias voltages.

structure of $\mathrm{Si}_{0.5} \mathrm{Ge}_{0.5} / \mathrm{Si} \mathrm{SL}$ can further increase the critical thickness of $\mathrm{Si}_{0.5} \mathrm{Ge}_{0.5}$ alloy on $\mathrm{Si}$ substrate ${ }^{12}$ and improve the optical confinement in our demonstrated structure. The $p$-type-doped profile and small thickness of the barrier and well $\left(33 \AA\right.$ ) of the $\mathrm{Si}_{0.5} \mathrm{Ge}_{0.5} / \mathrm{Si}$ SL can minimize the holetrapping problem in the traditional $\mathrm{Si} / \mathrm{SiGe}$ multiple quantum well (MQW). ${ }^{12,13} \mathrm{We}$, thus, adopted $p$-doped SL and a depleted Si layer to construct our photoabsorption region, which is similar to the structure of a $p-i-n$ photodiode with a partially $p$-doped photoabsorption region, ${ }^{14}$ for the purpose of achieving high saturation power ${ }^{14}$ and releasing the $R C$ bandwidth limitation due to the thin multiplication layer $(150 \mathrm{~nm})$ in our structure. ${ }^{11}$

The epitaxial layer structures were grown through ultrahigh vacuum/chemical vapor deposition (UHV/CVD) on an $n^{+}$-doped Si substrate. We fabricated the demonstrated device by using the standard photolithography, metallization, liftoff, and dry etching processes. As shown in Fig. 1(b), a coplanar waveguide (CPW), connected to the active area of the photodiodes, was fabricated on passivated $\mathrm{Si}_{3} \mathrm{~N}_{4}$ film for high-speed measurement. Finally, the fabricated devices were carefully cleaved for edge illumination, but no antireflection coating layers were applied to the facets.

We employed a mode-locked Ti:sapphire laser, which has a center wavelength of $830 \mathrm{~nm}$, as the light source for dc photocurrent and ac transient measurements. An electrical sampling scope with a $50 \mathrm{GHz}$ electrical bandwidth was also used to measure the impulse responses of the device. ${ }^{5-8} \mathrm{We}$ fabricated devices with different waveguide widths and lengths, and the device absorption length and waveguide width for the complete photoabsorption process were around 20 and $2 \mu \mathrm{m}$, respectively. We used a typical objective lens with a 0.5 numerical aperture (NA) to focus the injected light with a $2 \mathrm{~mm}$ beam diameter on the facets of the devices. Figure 2 shows the measured dc photocurrent and full width at half maximum (FWHM) of the measured impulse responses versus the reverse dc bias voltage under fixed optical power excitation $(0.225 \mathrm{~mW})$. The waveguide width and length of the measured devices were 2 and $60 \mu \mathrm{m}$, respectively. The inset shows the measured total current (dark current added photocurrent), the operation gain, and the dark current versus the reverse bias voltage. We determined the Downloaded 13 Feb 2009 to 140.112 .113 .225 . Redistribution subje

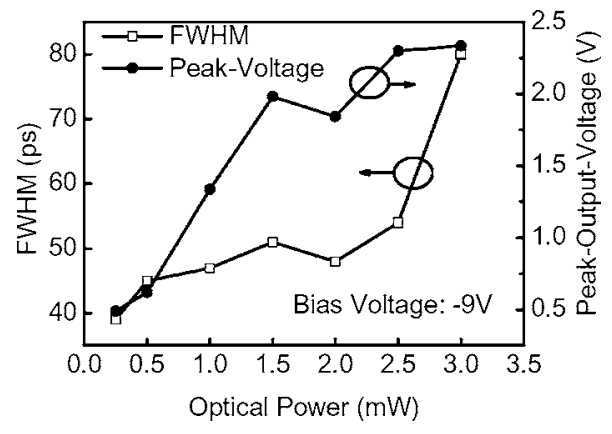

FIG. 3. FWHM (open square) and peak output voltages (closed circle) of the measured impulse responses vs the optical pumping power.

theoretical responsivity under unity gain operation by using the value of the photocurrent under $-9 \mathrm{~V}$ bias, which is the bias voltage required to saturate the drift velocity of photogenerated carriers, after correcting the coupling $(-3 \mathrm{~dB})$ loss. Under $-25 \mathrm{~V}$ bias, the avalanche gain was around 4.2, and the measured FWHM did not exhibit significant broadening. High bandwidth-efficiency product performance of this device could thus be expected. Figure 3 shows the corrected peak output voltage and measured FWHM of the impulse responses versus the optical pumping power under a fixed dc bias voltage $(-9 \mathrm{~V})$. We deembedded the microwave-loss effects of the bias tee and cable on peak output voltages using a radio-frequency (rf) power sensor and a pattern generator, which could generate electrical pulses with 50 ps FWHM and mimic the operation of our device under optical pulse illumination. The values of the corrected peak output voltages were around three times higher than the measured values. The measured FWHM of the impulse response did not broaden significantly when the average optical power reached $2 \mathrm{~mW}$. By utilizing the fast-Fourier-transform (FFT) technique and the measured impulse responses, we were able to estimate the bandwidth performance of our device under different levels of optical power excitation. ${ }^{5-8}$ In FFT calculation, we expanded the window of the integration time to $1.5 \mathrm{~ns}$, which is larger than the fitted decay time constant of traces under high-power excitation ( $2 \mathrm{~mW}, 1.2 \mathrm{~ns})$, and did not omit the long tail of high-power traces in order to enhance the resolution and precision of our transformed frequency response. Traces A and B in Fig. 4 represent the measured impulse responses under 0.225 and $2 \mathrm{~mW}$ optical power excitations, respectively. The solid line and dotted line in the inset represent the calculated frequency response with and without deembedding of the influence of the bias tee, cable, and microwave probe, respectively. Under low-power excitation $(0.225 \mathrm{~mW})$, the obtained $f_{3 \mathrm{~dB}}$ bandwidths ( 9 and $13 \mathrm{GHz}$ ) and external quantum efficiency (31.5\%) of our device were comparable to those of Si-based RCE PDs, 6,7 which exhibit serious wavelength selectivity, in the $800 \mathrm{~nm}$ wavelength regime. For the case of high optical power excitation, the FWHM of the measured trace broadened slightly (48 ps), and a long tail with a decay time constant of around 1.2 ns was observed. The observed tail can possibly be attributed to the diffusion current in the $p$ - or $n$ - doped regions. ${ }^{6,7}$ The transformed $f_{3 \mathrm{~dB}}$ bandwidth was around $4 \mathrm{GHz}$ and reached $6 \mathrm{GHz}$ after the influence of the measurement system was deembedded. The output saturation current under continuous-wave (cw) operation and the peak output current (voltage) under pulse-mode operation are two to AlP license or copyright; see http://apl.aip.org/apl/copyright.jsp 


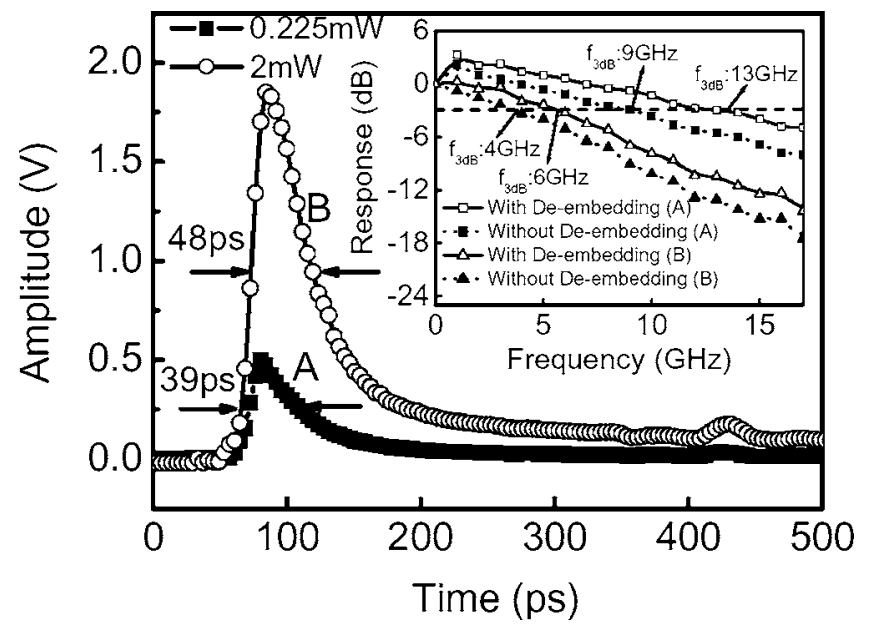

FIG. 4. The measured impulse responses of the device under different levels of optical power excitation and a fixed dc bias voltage of $-9 \mathrm{~V}$. Trace A: $0.225 \mathrm{~mW}$. Trace B: $2 \mathrm{~mW}$. The inset shows the corresponding frequency responses with and without deembedding of the influence of the measurement system.

FOMs that are usually used to evaluate the performance of high-power photodiodes. ${ }^{10,15,16}$ As shown in Fig. 4, the obtained peak output voltages of these two traces, even without correcting the rf loss $(0.17 \mathrm{~V}$ for trace $\mathrm{A}$ and $0.65 \mathrm{~V}$ for trace $\mathrm{B})$, were still much higher than the reported values of peak output voltages of Si-based RCE PDs ( $~ 80 \mathrm{mV})$ (Ref. 6) and Ge-on-SOI PDs (Ref. 8) $(\sim 25 \mathrm{mV})$ under high-speed operation. Although the peak-output-voltage values of the reported photodiodes can be increased by increasing the optical pumping power, their speed performance may be seriously degraded under high optical power illumination even by increasing the dc bias voltage, which is usually observed in the ordinary $p-i$ - $n$ photodiode. ${ }^{16}$ Figure 5 and its inset show the measured impulse response and the corresponding frequency response of the device in the avalanche-operation

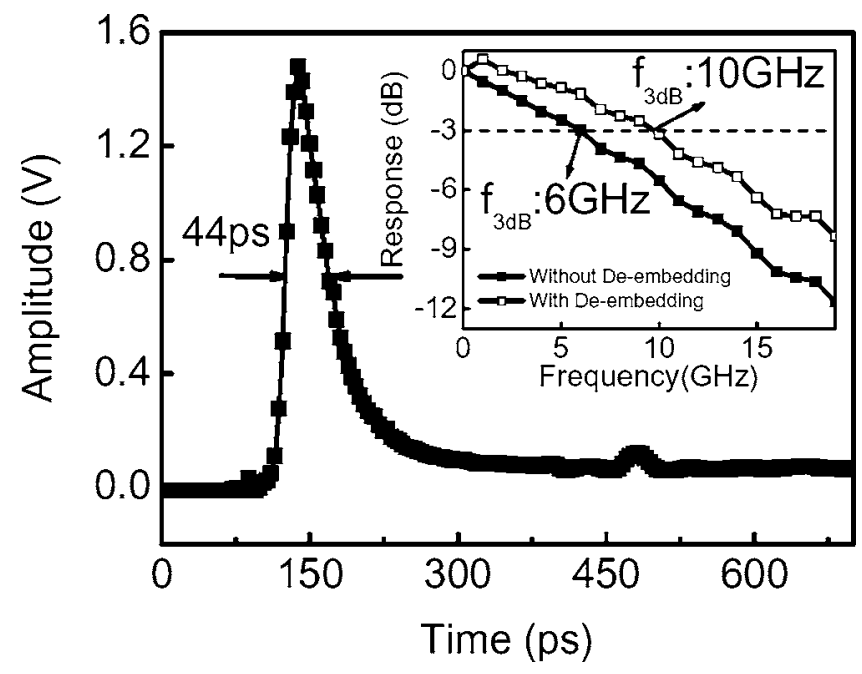

FIG. 5. The measured impulse responses under a $-25 \mathrm{~V}$ bias voltage and a fixed optical pumping power $(0.225 \mathrm{~mW})$. The inset shows the corresponding frequency responses with and without deembedding of the influence of the measurement system. regime $(-25 \mathrm{~V})$, respectively. The illuminated optical power was $0.225 \mathrm{~mW}$. According to the measurement results shown in Figs. 2 and 5, our demonstrated edge-coupled photodiode can achieve extremely high external efficiency (276\%, $1.85 \mathrm{~A} / \mathrm{W})$, wide electrical bandwidth $(10 \mathrm{GHz}$ after correcting for the influence of the system), and high peak output voltage $(1.5 \mathrm{~V})$ simultaneously when operating in the avalanche mode. The achieved bandwidth-efficiency product $(27.6 \mathrm{GHz}, 10 \mathrm{GHz}, 276 \%)$ and bandwidth-peak-outputvoltage product $(15 \mathrm{GHz} \mathrm{V}, 10 \mathrm{GHz}, 1.5 \mathrm{~V})$ are both excellent as compared to the performances of reported siliconbased photodiode under linear (with $46 \%$ efficiency and $29 \mathrm{GHz}$ corrected bandwidth ${ }^{8}$ ) or avalanche-operation regime (with internal gain equals to $10,0.8 \mathrm{~A} / \mathrm{W}$ responsivity, and $8 \mathrm{GHz}$ bandwidth). ${ }^{3}$ The demonstrated promising results for our device verify its usefulness for $10 \mathrm{Gbyte} / \mathrm{s}$ shortreach optical fiber communications.

In conclusion, we have demonstrated a Si/SiGe-based photodiode operated in the $830 \mathrm{~nm}$ wavelength regime. High bandwidth-efficiency product performance $(27.6 \mathrm{GHz})$ and high bandwidth-peak-output-voltage product performance $(15 \mathrm{GHz} \mathrm{V})$ have been achieved simultaneously under avalanche operation.

This work was sponsored by the National Science Council of Taiwan under Grant No. NSC-95-2215-E-008-003 and by the Department of Industrial Technology, the Ministry of Economic Affairs under Grant No. 93-EC-17-A-07-S1-0001.

${ }^{1}$ S. M. Csutak, J. D. Schaub, W. E. Wu, and J. C. Campbell, IEEE Photonics Technol. Lett. 14, 516 (2002).

${ }^{2}$ E. Sciacca, A. Giudice, D. Sanfilippo, F. Zappa, S. Lombardo, R. Consentino, C. Di Franco, M. Ghioni, G. Fallica, G. Bonanno, S. Cova, and E. Rimini, IEEE Trans. Electron Devices 50, 918 (2003).

${ }^{3}$ B. Yang, J. D. Schaub, S. M. Csutak, D. L. Rogers, and J. C. Campbell, IEEE Photonics Technol. Lett. 15, 745 (2003).

${ }^{4}$ M. Yang, K. Rim, D. L. Rogers, J. D. Schaub, J. J. Welser, D. M. Kuchta, D. C. Boyd, F. Rodier, P. A. Rabidoux, J. T. Marsh, A. D. Ticknor, Q. Yang, A. Upham, and S. C. Ramac, IEEE Electron Device Lett. 23, 395 (2002).

${ }^{5}$ H. C. Lee and B. V. Zeghbroeck, IEEE Electron Device Lett. 16, 175 (1995).

${ }^{6}$ M. K. Emsley, O. Dosunmu, and M. S. Unlu, IEEE J. Sel. Top. Quantum Electron. 8, 948 (2002)

${ }^{7}$ M. K. Emsley, O. Dosunmu, and M. S. Unlu, IEEE Photonics Technol. Lett. 14, 519 (2002).

${ }^{8}$ G. Dehlinger, S. J. Koester, J. D. Schaub, J. O. Chu, Q. C. Ouyang, and A. Grill, IEEE Photonics Technol. Lett. 16, 2547 (2004).

${ }^{9}$ M. Jutzi, M. Berroth, G. Wohl, M. Oehme, and E. Kasper, IEEE Photonics Technol. Lett. 17, 1510 (2005).

${ }^{10}$ K. Kato, IEEE Trans. Microwave Theory Tech. 47, 1265 (1999).

${ }^{11}$ J.-W. Shi, Y.-H. Liu, and C.-W. Liu, J. Lightwave Technol. 22, 1583 (2004).

${ }^{12}$ D. Buca, S. Winnerl, S. Lenk, Ch. Buchal, and D.-X. Xu, Appl. Phys. Lett. 80, 4172 (2002)

${ }^{13}$ C. Li, Q. Yang, H. Wang, J. Yu, Q. Wang, Y. Li, J. Zhou, H. Huang, and X. Ren, IEEE Photonics Technol. Lett. 12, 1373 (2000).

${ }^{14}$ X. Li, N. Li, S. Demiguel, X. Zheng, J. C. Campbell, H. H. Tan, and C. Jagadish, IEEE Photonics Technol. Lett. 16, 2326 (2004).

${ }^{15}$ J.-W. Shi, K. G. Gan, Y.-H. Chen, C.-K. Sun, Y. J. Chiu, and John E. Bowers, IEEE Photonics Technol. Lett. 14, 1587 (2002).

${ }^{16}$ P.-L. Liu, K.-J. Williams, M. Y. Frankel, and R. D. Esman, IEEE Trans. Microwave Theory Tech. 47, 1297 (1999). 